\title{
Immune reconstitution inflammatory syndrome in HIV and sporotrichosis coinfection: report of two cases and review of the literature
}

\author{
Marcelo Rosandiski Lyra ${ }^{[1],[2],}$ Maria Letícia Fernandes Oliveira Nascimento ${ }^{[3],}$ \\ Andréa Gina Varon ${ }^{[4]}$, Maria Inês Fernandes Pimentel ${ }^{[1]}$, Liliane de Fátima Antonio ${ }^{[1]}$, \\ Maurício Naoto Saheki ${ }^{[1]}$, Sandro Javier Bedoya-Pacheco ${ }^{[1]}$ \\ and Antonio Carlos Francesconi do Valle ${ }^{[1]}$
}

[1]. Laboratório de Vigilância em Leishmanioses, Instituto Nacional de Infectologia Evandro Chagas, Fundação Oswaldo Cruz, Rio de Janeiro, RJ. [2]. Laboratório de Pesquisa Clínica em Dermatologia Infecciosa, Instituto Nacional de Infectologia Evandro Chagas, Fundação Oswaldo Cruz, Rio de Janeiro, RJ. [3]. Departamento de Doenças Infecciosas, Hospital Central do Exército, Rio de Janeiro, RJ. [4]. Laboratório de Micobactéria, Instituto Nacional de Infectologia Evandro Chagas, Fundação Oswaldo Cruz, Rio de Janeiro, RJ.

\begin{abstract}
We report 2 cases of patients with immune reconstitution inflammatory syndrome (IRIS) associated with cutaneous disseminated sporotrichosis and human immunodeficiency virus (HIV) coinfection. The patients received specific treatment for sporotrichosis. However, after 4 and 5 weeks from the beginning of antiretroviral therapy, both patients experienced clinical exacerbation of skin lesions despite increased T CD4+ cells (T cells cluster of differentiation 4 positive) count and decreased viral load. Despite this exacerbation, subsequent mycological examination after systemic corticosteroid administration did not reveal fungal growth. Accordingly, they were diagnosed with IRIS. However, the sudden withdrawal of the corticosteroids resulted in the recurrence of IRIS symptoms. No serious adverse effects could be attributed to prednisone. We recommend corticosteroid treatment for mildto-moderate cases of IRIS in sporotrichosis and HIV coinfection with close follow-up.
\end{abstract}

ABSTRACT

Keywords: HIV. Sporotrichosis. Immune reconstitution inflammatory syndrome.

\section{INTRODUCTION}

Sporotrichosis is a cosmopolitan subcutaneous mycosis caused by Sporothrix spp. fungal infection ${ }^{1}$. Infection is generally acquired through skin trauma during handling of soil or plants. In the last 20 years, transmission by infected cats has been increasingly reported in the State of Rio de Janeiro, Brazil, and the main causative species is Sporothrix brasiliensis ${ }^{1}$. The Instituto Nacional de Infectologia Evandro Chagas (INI/FIOCRUZ) is a regional reference center for the diagnosis and treatment of sporotrichosis, with over 1,848 human cases reported between 1997 and $2007^{2}$. The number of new cases in the State of Rio de Janeiro remains high, and no decrease is forecasted in the short term.

Address to: Enfa. Liliane de Fátima Antonio. Lab VigiLeish/INI/FIOCRUZ. Av. Brasil 4365, Manguinhos, 21040-360 Rio de Janeiro, RJ, Brasil. Phone: 5521 9-8022-8384; 5521 3865-9541

e-mail: lilianedefatima@gmail.com; liliane.fatima@ini.fiocruz.br

Received 16 June 2014

Accepted 14 October 2014
The worsening of skin lesions or appearance of new lesions during the treatment of sporotrichosis and human immunodeficiency virus (HIV) may suggest immune reconstitution inflammatory syndrome (IRIS). However, few such cases have been reported, and all were HIVpositive patients already on antifungal treatment who started antiretroviral treatment and experienced clinical exacerbation with reactivation and/or emergence of new lesions in previous scars or spared areas $^{3,4}$.

We report 2 cases of IRIS in patients co-infected with HIV and sporotrichosis at INI/FIOCRUZ that were successfully treated with systemic corticosteroids.

\section{CASE REPORT}

Case 1

A 23-year-old man was referred to the dermatology department with a diagnosis of disseminated cutaneous sporotrichosis refractory to irregular treatment with itraconazole $200 \mathrm{mg}$ daily. Several painful ulcerated lesions with seropurulent discharge were distributed on the trunk, and upper and lower limbs. Mycological examination of samples collected from the lesions confirmed sporotrichosis, and HIV serology was positive. 
The initial TCD4+ (T lymphocyte cluster of differentiation 4 positive) count and viral load were 43 cells $/ \mathrm{mm}^{3}$ and 21,261 copies $/ \mathrm{mL}$, respectively. After 4 months of itraconazole $200 \mathrm{mg}$ daily, the skin lesions partially healed (Figure 1). However, 4 weeks after the introduction of highly active antiretroviral therapy (HAART), the lesions recurred in association with poor general condition, prostration, high fever, arthralgia, and myalgia. The skin lesions worsened (Figure 2) and became more painful than during their onset. New serum tests showed a CD4 $4^{+}$count of 54 cells $/ \mathrm{mm}^{3}$ and a viral load $<50$ copies $/ \mathrm{mL}$. Cultures of cerebrospinal fluid and a fragment of a skin lesion were both negative for fungi. After hospitalization, itraconazole was replaced by $50 \mathrm{mg}$ of amphotericin B deoxycholate plus prednisone $40 \mathrm{mg}$ daily. The patient's general condition and involution of skin lesions improved rapidly. After a cumulative dose of 700mg amphotericin B deoxycholate, itraconazole $200 \mathrm{mg}$ daily was restarted. However, a new episode of IRIS with clinical deterioration occurred 3 months after prednisone suspension, and this drug was then re-introduced. Progressive prednisone withdrawal took 10 months, and the patient remained on itraconazole $200 \mathrm{mg}$ daily for 18 months until the lesions healed completely. After the termination of the treatment, the patient presented with undetectable viral load and a $\mathrm{CD} 4^{+}$count of 309 cells $/ \mathrm{mm}^{3}$.

\section{Case 2}

A 37-year-old man was referred to the dermatology department presenting with a 1-month clinical evolution of multiple ulcers and necrotic lesions scattered over the face (Figure 3), upper limbs, neck, and trunk in association with poor general condition. Rapid test for HIV serology was positive, and mycological examination of a sample collected from a lesion confirmed disseminated cutaneous sporotrichosis. Therefore, the patient was treated with amphotericin B deoxycholate (cumulative dose, $650 \mathrm{mg}$ ) for 2 weeks followed by itraconazole $200 \mathrm{mg}$ daily. $\mathrm{CD}^{+}$count and viral load were 66 cells $/ \mathrm{mm}^{3}$ and 423,153 copies/mL. HAART was initiated on day 10 of antifungal therapy. Two weeks later, the patient's general condition substantially improved and the skin lesions partially regressed. However, on the fifth week of antiretroviral therapy, his general condition worsened, with high fever, arthritis in the knees and elbows, increased phlogosis in old lesions, and the appearance of small new lesions. Serum samples showed a $\mathrm{CD} 4^{+}$count of 95 cells $/ \mathrm{mm}^{3}$ and a viral load of 21,303 copies/ $\mathrm{mL}$. Cultures of samples from the skin lesions were negative for fungi. He received prednisone $40 \mathrm{mg}$ /day and showed improvement after 48 hours, with no fever or arthralgia as well as decreased inflammation of the skin lesions. There was no need to suspend HAART or reintroduce amphotericin B. The lesions healed after itraconazole $200 \mathrm{mg}$ daily for an additional 12 months. The dose of prednisone was gradually reduced and was discontinued after 10 months of treatment. After the discontinuation of itraconazole, the patient had undetectable viral load and a CD4 $4^{+}$count of 56 cells $/ \mathrm{mm}^{3}$.

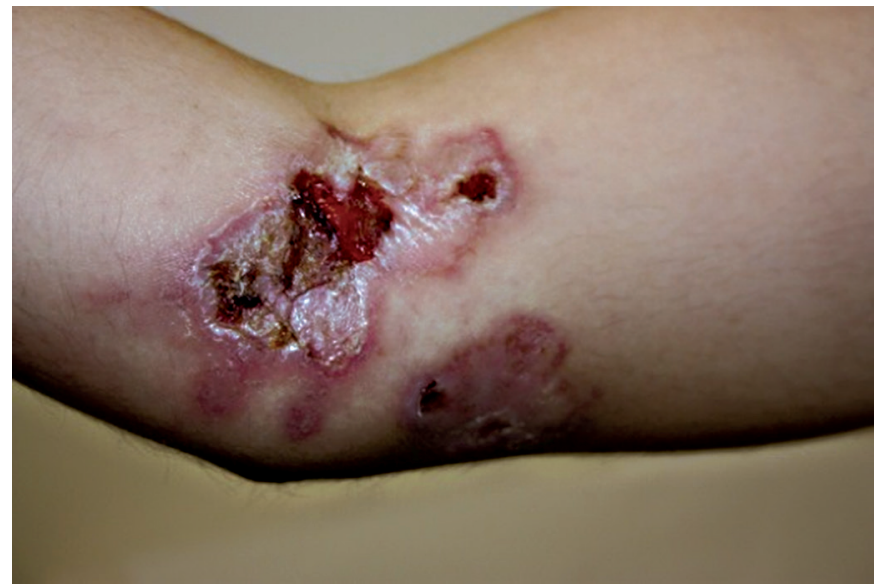

FIGURE 1 - Case 1: Ulcerative lesions on the upper limbs during itraconazole therapy before HAART. HAART: highly active antiretroviral therapy.

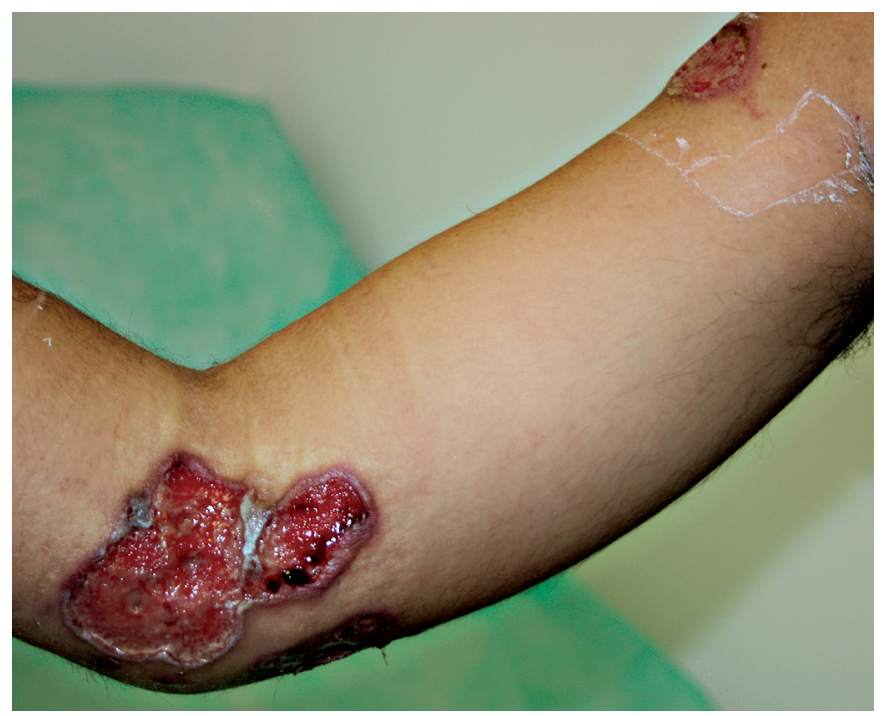

FIGURE 2 - Case 1: Same lesion in Figure 1 after starting HAART. HAART: highly active antiretroviral therapy.

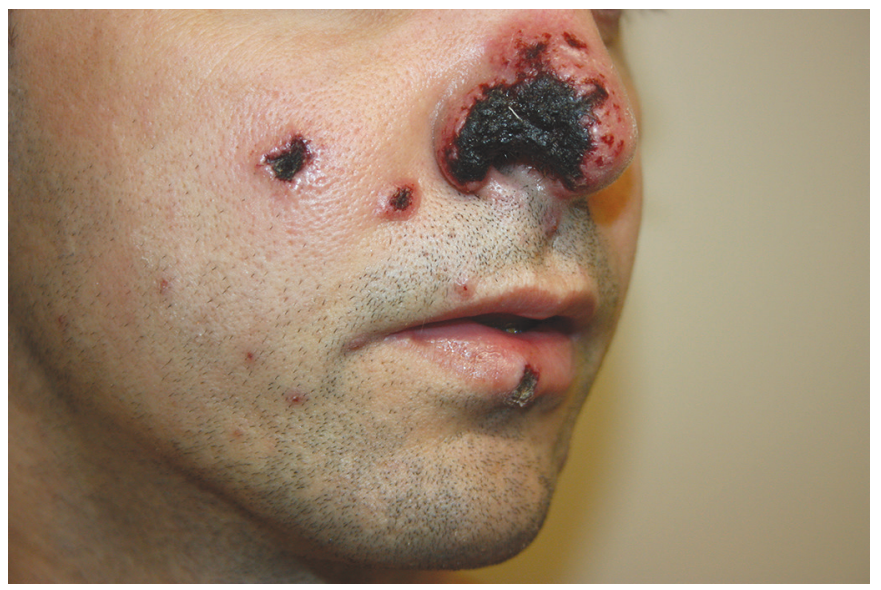

FIGURE 3 - Case 2: Ulcero-necrotic lesions on the face. 


\section{DISCUSSION}

About $83 \%$ of sporotrichosis patients have previous contact with infected cats 5 . Sporotrichosis is endemic to Rio de Janeiro and is associated with the HIV pandemic, has resulted in the emergence of cases of HIV and sporotrichosis coinfection. Coinfected individuals usually manifest disseminated mycosis with subcutaneous nodules, which may progress to suppuration and/or ulceration, probably resulting from hematogenous spread from a primary focus ${ }^{6}$.

Immune reconstitution inflammatory syndrome is defined as an exacerbation of a previous condition during the resolution process that occurs during the early period of HAART. It is caused by cellular hyperactivity that occurs with rapid numerical and functional recovery of $\mathrm{CD}^{+} \mathrm{T}$ lymphocyte counts, consequently increasing inflammatory reaction and damage to tissues, which can endanger the patient's life. Although there is no specific diagnostic laboratory test for IRIS, predictive risk factors for this disease include high viral load concentration during opportunistic infection, low $\mathrm{CD}^{+}$count at the beginning of HAART (i.e., $<200$ cells $/ \mathrm{mm}^{3}$ ), initiation of HAART shortly after the diagnosis of an opportunistic infection, and a rapid decrease in viral load with treatment ${ }^{7}$.

Both patients reported herein were first diagnosed with disseminated cutaneous sporotrichosis. The extent of infection lead to the suspicion of immunodeficiency, and HIV infection was diagnosed soon after. Both patients were already in an advanced state of immunosuppression, with $\mathrm{CD}^{+}$counts $<100$ cells $/ \mathrm{mm}^{3}$. The patients started antifungal therapy followed by antiretroviral treatment. Both patients improved with the regression of lesions. However, 4 and 5 weeks after the start of HAART, both patients exhibited paradoxical clinical worsening. Their clinical presentations were more consistent with IRIS than infection progression or fungal resistance to itraconazole.

In Rio de Janeiro, cases of HIV and sporotrichosis coinfection are increasingly common. However, few cases of IRIS related to this mycosis have been reported. The only cases were reported in 2010 , including 2 cases of central nervous system disease ${ }^{3}$ and 2 cases of cutaneous lymphatic disease ${ }^{4}$; the latter 2 cases had cultures positive for $S$. schenckii from fragments obtained by skin lesion biopsy. However, in both cases reported herein, the first fungi cultures were positive, and the subsequent ones after treatment were negative. A review of 196 patients with different opportunistic infections revealed 21 IRIS cases occurring after the start of HAART ${ }^{8}$. Neurocryptococcosis is the predominant fungal infection in IRIS. In such cases, the culture results remain negative during IRIS 9 .

The present patients experienced lesion recurrence as well as the appearance of new lesions along with systemic inflammatory symptoms such as fever and arthralgia. The increase in CD4 ${ }^{+}$ count and rapid decrease in viral load are mandatory criteria for differentiating IRIS from disease progression or fungal resistance to antimycotic agents. Both patients were treated with prednisone shortly after IRIS diagnosis, resulting in rapid improvement of pain symptoms, fever defervescence, and involution of skin lesions. The use of corticosteroids to treat IRIS is controversial; some authors recommend their use only in severe cases $^{10}$, while others recommend listening to expert opinions ${ }^{11}$. The risks and benefits of corticosteroids for the treatment of non-life-threatening cases of IRIS are poorly understood. There is also no clear definition of low or moderate intensities of IRIS. Furthermore, there is no consensus on the use of corticosteroids for the control of the disease, which can lead to serious adverse events including infectious and metabolic events. Neither patient in this report was in critical condition. However, owing to the discomfort of the systemic inflammatory reaction, we opted to prescribe corticosteroids. Both patients exhibited rapid clinical improvement. However, the sudden withdrawal of the drug resulted in the recurrence of IRIS symptoms. Safe withdrawal of prednisone took several months in both cases, and no serious adverse effects that could be attributed to prednisone occurred.

No studies demonstrate survival improvement with the use of corticosteroids in IRIS caused by sporotrichosis. However, corticosteroid therapy provided rapid improvements in the symptoms and well being of the present patients, supporting their administration. However, long-term corticosteroid therapy in immunosuppressive doses can result in adverse effects. Thus, the lowest possible dose that induces clinical remission must be administered, and the treatment must be discontinued as soon as possible. It is important to note that as neither patient was at risk of death, we cannot evaluate the impact of corticosteroids on survival in severe cases. However, a randomized trial comparing prednisone with placebo in patients with IRIS associated with tuberculosis indicates prednisone treatment decreases hospitalization time, accelerates clinical recovery, and improves quality of life but does not decrease mortality ${ }^{12}$.

Both HIV and sporotrichosis are endemic in Rio de Janeiro, and sporotrichosis-related IRIS is a reality. The present patients received specific treatment for sporotrichosis followed by clinical deterioration after starting HAART; thereafter, they exhibited clinical improvement with negative cultures for fungi, typical of IRIS, and recovery after systemic corticosteroid administration. Therefore, we recommend corticosteroid treatment for mild-to-moderate cases of IRIS in sporotrichosis and HIV coinfection with close follow-up.

\section{REFERENCES}

1. Almeida-Paes R, Oliveira MM, Freitas DF, Valle AC, ZancopéOliveira RM, Gutierrez-Galhardo MC. Sporotrichosis in Rio de Janeiro, Brazil: Sporothrix brasiliensis is associated with atypical clinical presentations. PLoS Negl Trop Dis 2014; 8:e3094.

2. Silva MB, Costa MM, Torres CC, Galhardo MC, Valle AC, Magalhães MA, et al. Urban sporotrichosis: a neglected epidemic in Rio de Janeiro, Brazil. Cad Saude Publica 2012; 28:1867-1880.

3. Galhardo MC, Silva MT, Lima MA, Nunes EP, Schettini LE, Freitas RF, et al. Sporothrix schenckii meningitis in AIDS during immune reconstitution syndrom. J Neurol Neurosurg Psychiatry 2010; 81:696-699.

4. Gutierrez-Galhardo MC, Valle AC, Fraga BL, Schubach AO, Hoagland BR, Monteiro PC, et al. Disseminated sporotrichosis as 
a manifestation of immune reconstitution inflammatory syndrome. Mycoses 2010; 53:78-80.

5. Schubach A, Barros MB, Wanke B. Epidemic sporotrichosis. Curr Opin Infect Dis 2008; 21:129-133.

6. Heller HM, Fuhrer J. Disseminated sporotrichosis in patients with AIDS: case report and review of the literature. AIDS 1991; 5:12431246.

7. Bonham S, Meya DB, Bohjanen PR, Boulware DR. Biomarkers of HIV immune reconstitution inflammatory syndrome. Biomark Med 2008; 2:349-361.

8. Achenbach CJ, Harrington RD, Dhanireddy S, Crane HM, Casper $\mathrm{C}$, Kitahata MM. Paradoxical immune reconstitution inflammatory syndrome in HIV-infected patients treated with combination antiretroviral therapy after AIDS-defining opportunistic infection. Clin Infect Dis 2012; 54:424-433.
9. Sungkanuparph S, Filler SG, Chetchotisakd P, Pappas PG, Nolen TL, Manosuthi W, et al. Cryptococcal immune reconstitution inflammatory syndrome after antiretroviral therapy in AIDS patients with cryptococcal meningitis: A prospective multicenter study. Clin Infect Dis 2009; 49:931-934.

10. French MA. HIV/AIDS: immune reconstitution inflammatory syndrome: A reappraisal. Clin Infect Dis 2009; 48:101-107.

11. Meintjes G, Lynen L. Prevention and treatment of the immune reconstitution inflammatory syndrome. Curr Opin HIV AIDS 2008; 3:468-476.

12. Meintjes G, Wilkinson RJ, Morroni C, Pepper DJ, Rebe K, Rangaka MX, et al. Randomized placebo-controlled trial of prednisone for paradoxical TB-associated immune reconstitution inflammatory syndrome. AIDS 2010; 24:2381-2390. 